\title{
Use of drug-eluting stents in older patients with CKD
}

The ability of drug-eluting stents to lower restenosis and revascularization rates has been demonstrated in randomized, controlled trials; however, these devices are often used in subsets of patients that have not been studied in clinical trials. A large observational study has now compared the safety and efficacy of drugeluting and bare-metal stents in older patients with chronic kidney disease (CKD) and demonstrated that use of drug-eluting stents in these patients is safe and associated with reduced rates of myocardial infarction and death in some patient subgroups.

The growing prevalence of CKD has contributed to an increase in the number of affected patients undergoing percutaneous coronary interventions
(PCIs). Despite this increase, the representation of patients with CKD in randomized, controlled trials of PCIs is poor. Moreover, reports of an increased risk of late stent thrombosis associated with the use of drug-eluting stents in patients with CKD have brought into question the safety of such devices in these patients. In the present study, Thomas Tsai and colleagues compared the safety and efficacy of drug-eluting and bare-metal stents in older patients with CKD. They used the American College of Cardiology National Cardiovascular Data Registry (NCDR) to identify 283,593 patients who were $\geq 65$ years of age and had received a stent. The researchers then classified the patients into five groups according to their estimated glomerular filtration rate and assessed outcomes using the Medicare Services national claims database. As Tsai explains, "the use of devices in subsets of

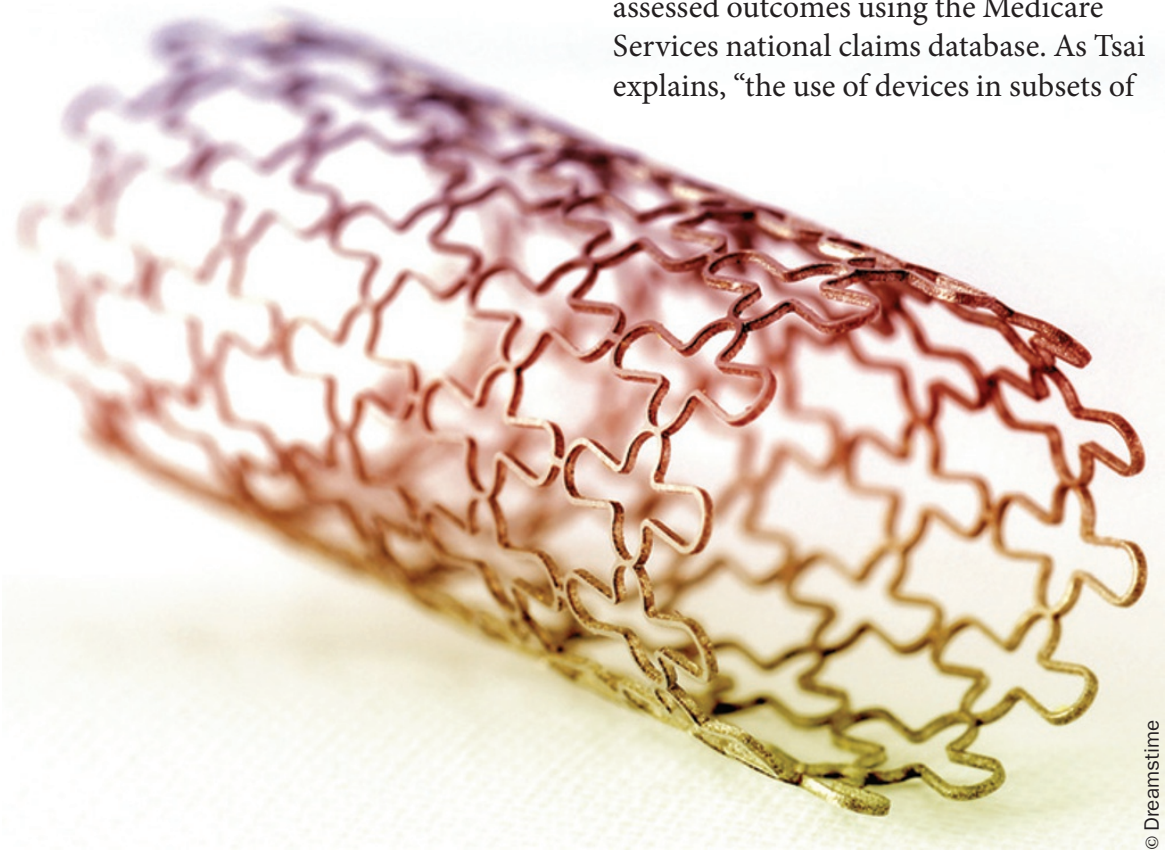

the population not represented in clinical trials is an ongoing reality. Comparative effectiveness registry work allows us to evaluate low frequency end points, such as generalized safety."

The researchers found that CKD was highly prevalent, with $>40 \%$ of patients having some form of CKD. Tsai et al. then compared outcomes associated with the use of drug-eluting and bare-metal stents. Compared with use of bare-metal stents, drug-eluting stents were associated with lower 30-month mortality rates in patients with normal renal function and in patients with CKD of any severity. Rates of myocardial infarction in patients with normal renal function or mild, moderate or severe CKD were lower in those who had received a drug-eluting stent than in those who had received a bare-metal stent. However, this association was not apparent in patients on long-term dialysis.

Tsai et al. say that the observed decrease in mortality rate and lack of notable safety hazards support the use of drugeluting stents in patients $>65$ years of age with CKD. "Because of the recent scare regarding the increased risk of late stent thrombosis in patients with $\mathrm{CKD}$ receiving a drug-eluting stent, it is comforting to know that there does not appear to be an increased risk of hard end points in a large cohort of patients receiving drug-eluting versus bare-metal stents," Tsai concludes.

Susan J. Allison

Original article Tsai, T. T. et al. Safety and efficacy of drugeluting stents in older patients with chronic kidney disease: a report from the linked CathPCI Registry-CMS Claims Database. J. Am. Coll. Cardiol. 58, 1859-1869 (2011) 